médecine/sciences $1987 ; 3: 608-12$

\section{Summary}

The two genetic complementation groups reported for Fanconi's anemia correspond to two phenotypic classes as characterized by measurements of the rate of DNA semiconservative synthesis after a DNA crosslinking treatment, i.e. the photoaddition of 8-methoxypsoralen. This test allows a rapid classification of Fanconi's anemia patients which appear to be a prerequisite for investigations of the biochemical defect(s) related to the processing of DNA interstrand crosslinks. A project aimed at the isolation of the genes involved in this disease was initiated in order to characterize the expression and regulation of the genes controlling sensitivity of human cells to DNA crosslinking agents. The conditions for the DNA-mediated transfer and expression of wild-type genes in Fanconi's anemia primary skin fibroblast cell lines were established. Restoration of a normal cellular resistance to a crosslinking treatment (mitomycin C) was achieved by transfection with high molecular weight DNA from normal human cells. The selection of spontaneous mytomycin Cresistant revertants was experimentally excluded. These experiments established the prerequisites for the isolation of the gene(s) involved and constitute one of the first successfull attempts to correct a human genetic disorder characterized by a defect in processing of DNA lesions.

\section{Remerciements}

Nous tenons à remercier Mmes C. Baroche, D. Chardonnieras et F. Sangrado pour leur excellente assistance. Ces travaux sont soutenus par le Cnrs (UA.533), l'Inserm (contrat $n^{\circ}$ 852017), le CEA, l'ARC et la CCE, Bruxelles (contrat $n^{\circ}$ BIO-151F)

\section{ADRESSE}

E. Moustacchi, C. Diatloff-Zito : institut Curie, biologie, 26, rue d'Ulm, 75231 Paris

\title{
L'anémie de Fanconi : aspects génétiques et moléculaires
}

\author{
Ethel Moustacchi, Catherine Diatloff-Zito
}

L

'anémie de Fanconi (AF) est une maladie autosomique récessive caractérisée par une pancytopénie et diverses malformations congénitales, principalement du squelette. L'anémie s'installe progressivement, généralement au cours de la première décennie de la vie [1]. De plus un pourcentage notable de malades développe une affection maligne, leucémique ou, moins souvent, tumorale. Cette maladie a été trouvée dans tous les groupes ethniques et la fréquence des hétérozygotes pour le gène $\mathrm{AF} a$ été estimée à 1:300 [2]. Chez ces derniers, la fréquence des affections malignes semble normale [3].

En 1964, Schroeder et al. [4] ont été les premiers à démontrer l'existence d'une fréquence élevée d'anomalies chromosomiques, essentiellement des cassures chromatidiennes et chromosomiques, dans les lymphocytes des malades. La fréquence de ces anomalies est notablement augmentée après traitement des lymphocytes in vitro par des agents de pontage interbrins de l'ADN. Il s'agit de drogues antitumorales telles que la mitomycine $\mathrm{C}$, le diépoxybutane, le cyclophosphamide, le cis-platine, les moutardes azotées bifonctionnelles, ou encore d'agents mutagènes tels que les psoralènes en combinaison avec une irradiation ultraviolette à $365 \mathrm{~nm}$. Cette augmentation des anomalies chromosomiques par rapport aux sujets normaux est considérée comme un signe essentiel du syndrome et sert au diagnostic prénatal [5] ainsi qu'à la détection de la maladie chez les patients pré-anémiques. Cette hypersensibilité chromosomique est accom- pagnée d'une hypersensibilité cellulaire à tous les agents de pontage de l'ADN, la sensibilité aux radiations ionisantes ou ultraviolettes de $254 \mathrm{~nm}$ restant proche de celle de cellules dérivées de donneurs normaux. Le ralentissement du cycle cellulaire, du fait d'un allongement de la phase G2, constitue également une caractéristique importante des lymphocytes $[6,7]$ ou des fibroblastes en culture dérivés de patients $A F$.

\section{Hétérogénéité génétique}

La variabilité dans le spectre des symptômes cliniques et de leur gravité [8], l'étalement dans le temps de l'apparition de la pancytopénie selon les patients et les différences de susceptibilité chromosomique et cellulaire in vitro aux agents pontants selon les lignées AF suggéraient l'existence d'une hétérogénéité génétique de cette maladie. En fait, la présence d'au moins deux groupes de complémentation génétique, $\mathrm{A}$ et $\mathrm{B}$, a été récemment mise en évidence par la technique d'hybridation somatique [9]. Brièvement, celleci consiste à faire fusionner in vitro des cellules dérivées de deux patients (sensibles à la mitomycine C par exemple) et à sélectionner les hybrides à l'aide de marqueurs génétiques appropriés. Si les propriétés des hybrides sont identiques à celles des cellules normales (résistantes à la mitomycine (C), on en déduit que les deux types parentaux sont porteurs de gènes différents puisqu'il y a complémentation des fonctions défectives dans les cellules des deux patients. En revanche, si les hybrides ont les mêmes proprié- 


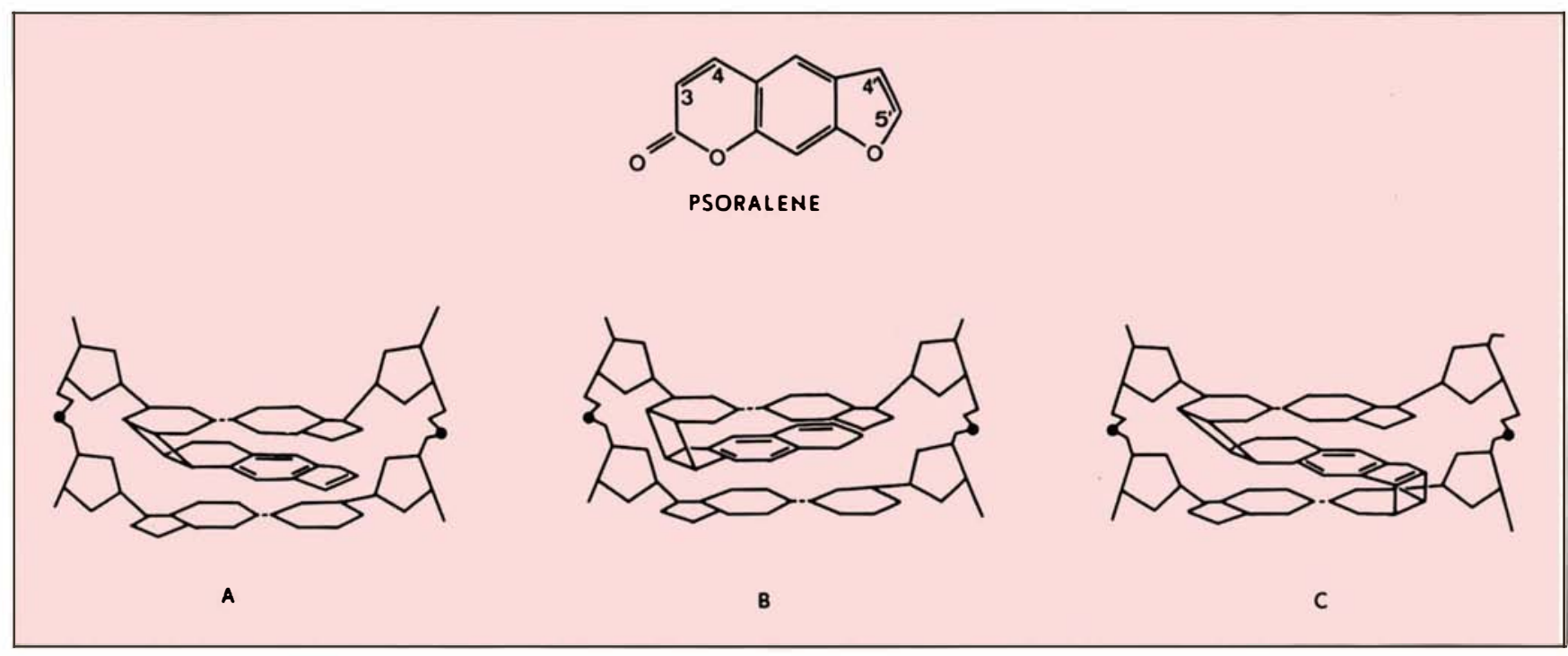

Figure 1. Interaction de molécules d'un psoralène avec de l'ADN après une irradiation à $365 \mathrm{~nm}$. A et $B$ correspondent aux deux types de monoadduits formés avec les pyrimidines. $C$ représente un pontage interbrin entre deux pyrimidines de I'ADN.

tés que les cellules parentales (sensibilité à la mitomycine $\mathrm{C}$ ), on en conclut qu'elles sont affectées dans le même gène. Certains des hybrides obtenus par fusion de cellules lymphoblastoïdes dérivées de patients AF différents ayant présenté une résistance à la mitomycine $\mathrm{C}$, l'existence d'au moins deux groupes de complémentation était prouvée.

Parallèlement il était démontré que phénotypiquement les lignées d'AF se rangeaient en deux classes vis-à-vis d'un paramètre cellulaire lié à la capacité de réparation des pontages interbrins de l'ADN, à savoir le taux de synthèse semi-conservative de l'ADN après un traitement pontant [10]. En effet, si des cellules sont traitées par du 8-méthoxypsoralène (8-MOP) en combinaison avec une irradiation à $365 \mathrm{~nm}$, on provoque la formation de mono-additions et de pontages entre les pyrimidines de l'ADN (figure 1). La présence de lésions entraîne une inhibition du taux de synthèse semi-conservative d'ADN dans les lignées normales, hétérozygotes ou AF. Cette phase d'inhibition est suivie d'une res$m / s n^{\circ} 10$ vol. 3, décembre 87

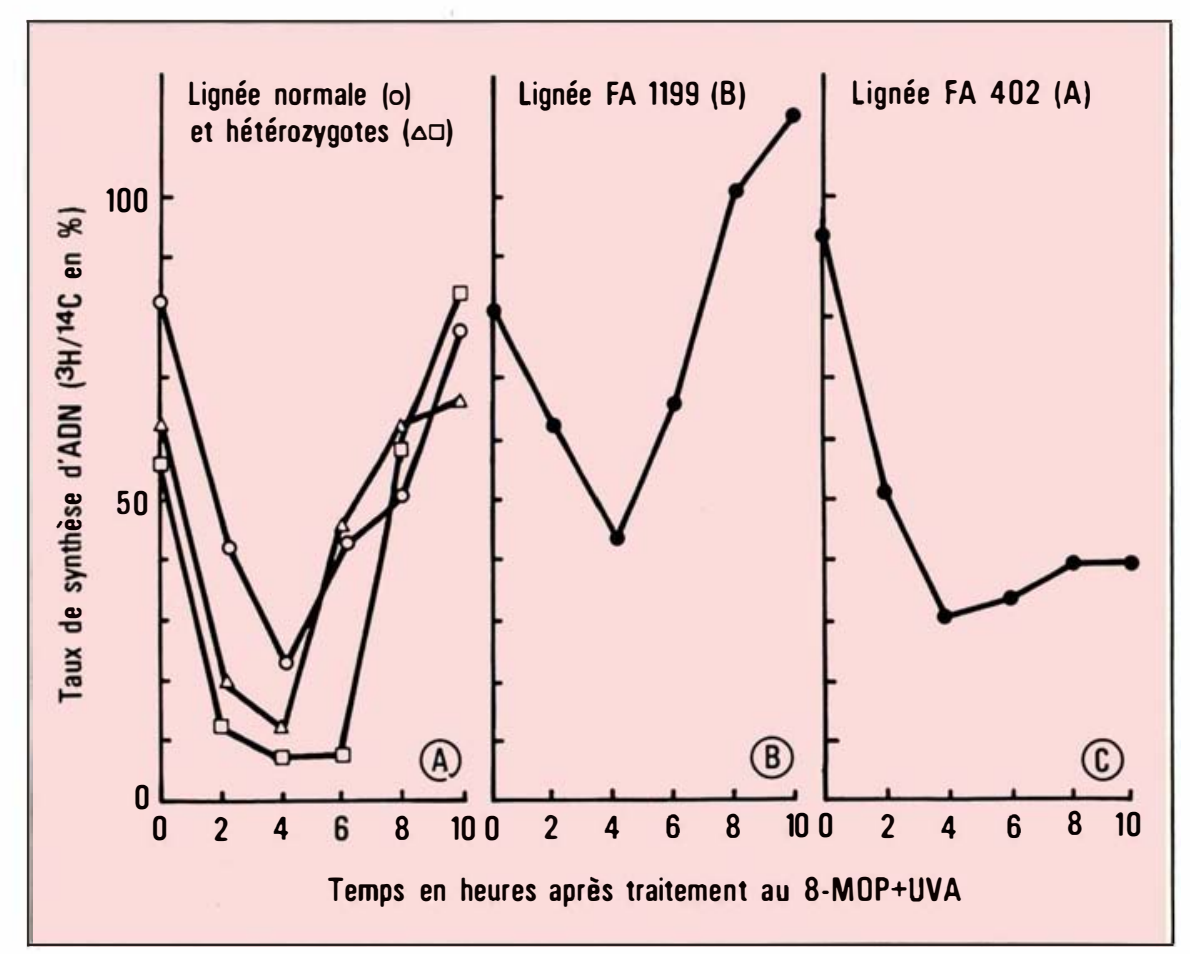

Figure 2. Taux de synthèse semi-conservative d'ADN dans des fibroblastos humains on fonction du temps après un traitement par lo 8-MOP (10-5 M) ot uno dose d'UVA (ultra-violots A) do $3,6 \mathrm{kJm}^{-2}$. (A) Une lignée normale (Jacquot) et deux lignées hétérozygotes $F A$ (F 311 et $F$ 253). (B) Une lignée FA appartenant au groupe de complémentation B d'après [9]. (C) Une lignée FA appartenant au groupe de complémentation $A d^{\prime}$ après les mêmes auteurs. Pour les détails techniques voir [10]. 
tauration d'un taux normal de synthèse associé à l'élimination des dommages dans les lignées normales ou hétérozygotes et dans une fraction des lignées $A F$ (figure $2 A$ et $2 B$ ). En revanche, une autre classe de lignées AF ne récupèrent pas un taux normal de synthèse d'ÂN (figure 2C). Par la suite, nous avons montré que les lignées $\mathrm{AF}$ appartenant au groupe de complémentation $B$, défini par hybridation somatique, sont celles qui présentent une capacité normale de restauration du taux de synthèse d'ADN alors que celles du groupe de complémentation A sont celles qui restent inhibées [11]. Ce test relativement rapide permet donc une classification des patients $\mathrm{AF}$ à partir de leurs fibroblastes dérivés de biopsies de peau ou de leurs lymphocytes transformés en lymphoblastes. Pour le moment, nous ne savons pas si l'appartenance aux groupes A ou B est reliée au degré de gravité de la maladie; une étude réalisée en collaboration avec le Dr E. Gluckman (hôpital Saint-Louis) est en cours. La stratégie thérapeutique, principalement constituée actuellement par la greffe de moelle, pourrait en effet être conditionnée en partie par la réponse à cette question.

Correction du défaut de l'AF par transfection avec de l'ADN de haut poids moléculaire de cellules normales

On ne sait rien jusqu'ici de l'expression et de la régulation des gènes affectés dans l'AF. Par ailleurs, l'obtention d'une sonde moléculaire serait extrêmement utile au diagnostic de cette maladie. Une fraction appréciable des patients présente en effet une partie seulement des symptômes cliniques et, dans certains cas, le test cytogénétique d'hypersensibilité chromosomique aux agents pontants n'est pas concluant. Aussi avons-nous entamé un programme de recherche qui vise au clonage des gènes impliqués dans
La première étape de ce travail a consisté à démontrer qu'il est possible de restaurer une réponse normale à un agent de pontage, tel que la mitomycine $\mathrm{C}$, par transfection de fibroblastes AF, par la technique de précipitation au phosphate de calcium, d'ADN de haut poids moléculaire obtenu à partir de cellules normales. Pour d'autres maladies génétiques caractérisées par une hypersensibilité aux radiations et à des agents chimiques, telles que le Xeroderma pigmentosum ou l'ataxie télangiectasie, les diverses tentatives de correction du défaut par transfection effectuées dans divers laboratoires se sont soldées par des échecs et ont souvent abouti à la sélection de mutations réverses de phénotype normal, préexistant spontanément à de faibles fréquences dans la population cellulaire ou étant induites par le traitement. Nous avons de ce fait été conduits à développer un protocole expérimental qui diffère par plusieurs points des protocoles précédemment adoptés. Ces différences peuvent se résumer ainsi.
- Utilisation de fibroblastes cutanés primaires non transformés afin d'éviter les remaniements chromosomiques liés à l'immortalisation par la transformation virale. Le nombre fini de passages possibles constitue bien entendu une limitation de ce choix. Il oblige à commencer la transfection à un niveau de passage relativement bas, compatible avec le nombre de générations requises pour toute la durée de l'expérience.

- Sélection de lignées $A F$ compétentes. La transfection est effectuée avec un mélange d'ADN de cellules humaines normales et d'un plasmide bactérien ( $\mathrm{pSV}_{2}$ neo) irradié aux ultraviolets* et contenant un gène de résistance aux antibiotiques sous contrôle d'un promoteur viral. Un gène bactérien sous contrôle d'un tel promoteur peut s'exprimer dans les cellules humaines et leur confere alors une résistance à l'antibiotique G418 (néomycine). Ce test effectué dans

* Le traitement du plasmide aux ultraviolets est connu pour augmenia la fréquence d'intégration stable du plasmide transfecté dans l'ADN de la cellule hôle $[12,13]$

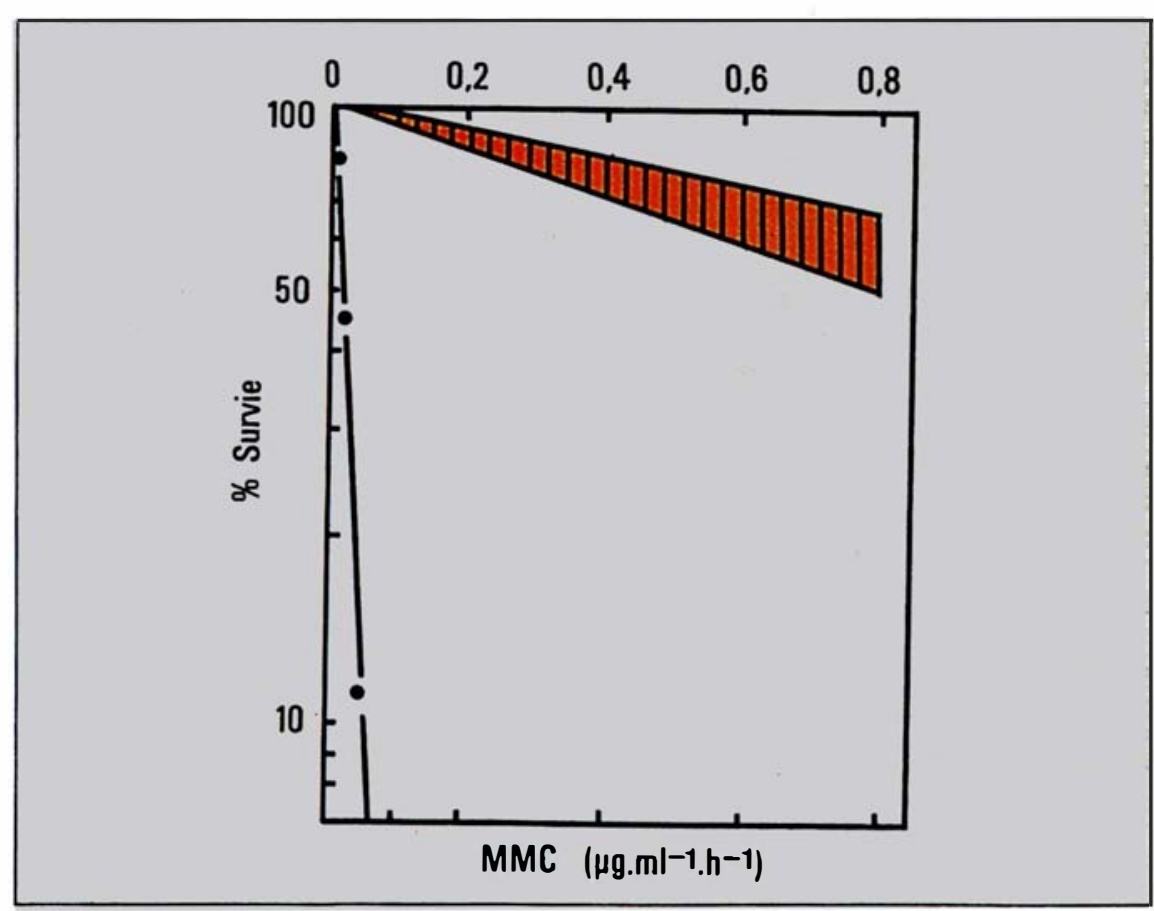

Figure 3. Survio en clonogénicité à la mitomycine C d’uno lignéo FA (FA 150) $(\bullet)$ et de plusieurs lignées normaies (zone hachurée). Pour les détails techniques, voir [14]. 


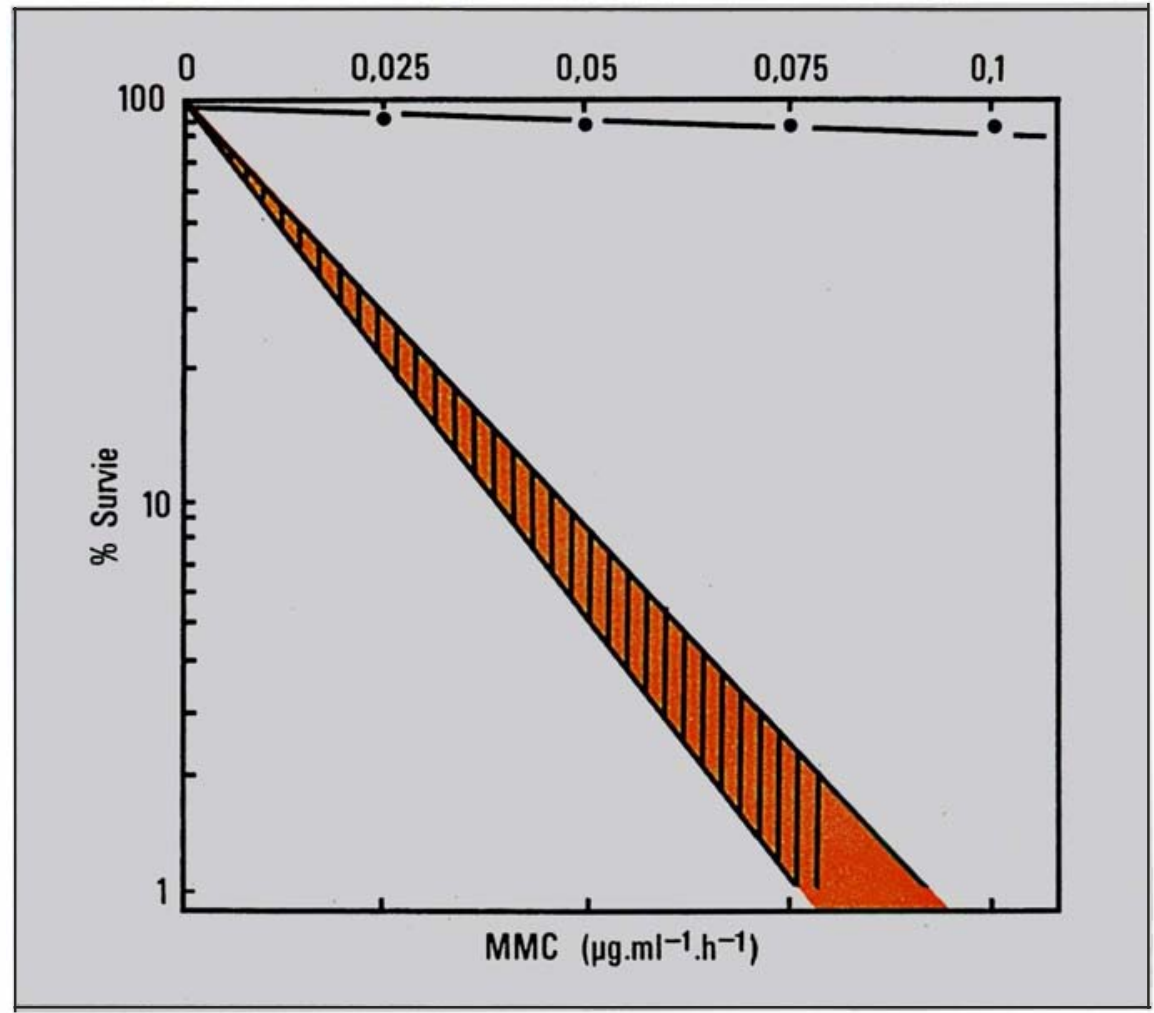

Figure 4. Survie après traitement à la mitomycine $C$ de fibroblastes de la lignée FA 150 transfectés avec de I'ADN normal de haut poids moléculaire (lignée TK6 MA). La survie à la MMC a été établie 40 jours après la transfection et sélection au $1^{\circ}$ jour par un traitement d'une heure de la population avec la mitomycine $C(0,025 \mu \mathrm{g} / \mathrm{ml})(\bullet)$. La zone hachurée correspond à la survie de la lignée FA 150 non transfectée ou transfectée avec son propre $A D N$ ou de l'ADN de levure ou de sperme de saumon ou encore soumise au protocole de transfection mais sans ADN. Pour les détails techniques, voir [14].

différentes souches $\mathrm{AF}$ permet de sélectionner des lignées AF compétentes, c'est-à-dire aptes à intégrer et à exprimer de l'ADN exogène $[12,13]^{*}$. C'est ainsi que, parmi trois lignées AF examinées, deux d'entre elles FA 150 et FA 121 se sont révélées compétentes pour la co-transfection par le plasmide irradié $\left(4 \times 10^{-6}\right.$ et $10^{-5}$, respectivement pour les fréquences de transfectants $n e o^{\mathrm{R}}$ ). En somme la sélection au G418 n'est appliquée, dans un premier temps, que pour caractériser les lignées AF pouvant être transformées par de l'ADN exogène. La co-transfection systématique des cellules par de l'ADN cellulaire total et le gène né $0^{R}$ nous sert par la suite à vérifier par Southern blot qu'il y a bien eu intégration d'ADN exogène dans les transformants examinés, transformants $m / s \quad n^{\circ} 10$ ool. 3, décembre 87 maintenue dans des conditions optimales de croissance de manière à favoriser le développement d'éventuels transformants de phénotype normal. Le temps de doublement plus court et la meilleure efficacité de clonage de ces derniers par rapport aux cellules $\mathrm{AF}$ permettent ainsi à la sélection naturelle de jouer en faveur de ces transformants. La figure 3 montre les courbes de survie à la mitomycine $\mathrm{C}$ de lignées normales et de la lignée FA 150 qui a servi dans les expériences ultérieures de transfection. On voit que les cellules de FA 150 sont environ 30 fois plus sensibles à ce produit que les cellules normales. La résistance à cet agent de pontage a été établie dans les cellules FA 15040 jours après la transfection et après 4 à 6 passages successifs en milieu de croissance. La figure 4 montre que la population a acquis la réponse typique d'une population normale. Il faut noter qu'au $11^{\circ}$ jour après la transfection, les cellules ont été soumises à un traitement d'une heure à la mitomycine $\mathrm{C}$ à $0,025 \mu / \mathrm{ml}$ de façon à éliminer une fraction notable (près de $80 \%$ ) des cellules AF sensibles à la mitomycine $\mathrm{C}$. La partie hachurée de la figure 4 représente l'ensemble des courbes de survie obtenues avec la lignée FA 150 non transfectée ou transfectée avec l'ADN de sperme de saumon ou de levure ou homologue (FA 150), ou encore soumises au protocole de transfection mais sans ADN. L'éventuelle présence de révertants mitomycine-résistants se traduirait sur ces courbes par une cassure. On voit que tel n'est pas le cas, ce qui permet d'exclure la sélection de mutants révertants dans les expériences de transfection positives.

Les courbes de survie de populations cellulaires comportant des proportions variables de cellules sensibles $\mathrm{AF}$ et de cellules résistantes normales $\left(10^{-2}\right.$ à $\left.10^{-4}\right)$, soumises à des concentrations croissantes de mitomycine $\mathrm{C}$ pendant une heure, présentent une rupture de pente (courbes à deux pentes). Ces courbes permettent d'estimer la fréquence des cellu- 
les résistantes à la mitomycine $\mathrm{C}$ dans les conditions qui miment une expérience de transfection. Compte tenu de cette fréquence, du nombre de cellules soumises à une transfection, du nombre de générations accomplies avant le traitement à la mitomycine $\mathrm{C}$, l'efficacité de transfert du marqueur de la résistance à la mitomycine $\mathrm{C}$ est estimée à $1-3 \times 10^{-7}$.

Les transfectants ont été caractérisés pour un ensemble de paramètres : détermination des antigènes HLA, pénétration de la mitomycine $C$ d'après la fréquence des pontages induits dans l'ADN, restitution du taux de la synthèse semi-conservative d'ADN après un traitement pontant (8-méthoxypsoralène et UVA), présence du marqueur neo [14]. Ces expériences permettent d'exclure une contamination par une autre lignée. Elles montrent que la fréquence de pontages est la même que dans une lignée normale éliminant ainsi la possibilité d'un défaut de pénétration de la drogue. Elles indiquent qu'au moins deux caractéristiques des lignées normales (survie à la mitomycine $\mathrm{C}$ et taux de synthèse d'ADN normal) sont corrigées. Elles démontrent enfin par Southern blot que l'ADN exogène (gène neo) est toujours présent dans les transfectants sous forme libre et intégrée. L'existence du gène $n e o^{R}$ sous une forme libre, plusieurs générations après la transfection, pourrait être due à des événements de recombinaison affectant la forme initialement intégrée du gène. Des exemples analogues ont été rapportés dans la littérature.

Les conditions préalables au clonage d'un gène, à savoir la complémentation du défaut de l'AF par un ADN humain normal, étant donc remplies, nous poursuivons actuellement cette recherche par l'étude de la correction par un ADN de souris. Celui-ci peut en effet être distingué de l'ADN humain à l'aide de séquences spécifiques de souris. Inversement, des mutants cellulai- logue aux cellules AF car spécifiquement sensibles aux agents de pontage sont disponibles. Nous tentons de corriger ce défaut des cellules de souris $M C^{s}$ par transfection avec de l'ADN humain normal. A ce jour, une seule famille de gènes humains, ERCC (excision repair complementing defective repair in Chinese hamster cells), impliqués dans la réparation des lésions UV-induites a été clonée. Ceci a été possible par correction de mutants UV-sensibles de hamster par transfection avec de l'ADN humain $[15,16]$. Notons toutefois que ces gènes ERCC ne complémentent pas le défaut de Xeroderma pigmentosum.

Conclusion. Avec le Xeroderma pigmentosum, l'ataxie télangiectasie et une demi-douzaine d'autres syndromes, l'anémie de Fanconi fait partie de cette classe de maladies génétiques rares prédisposant au cancer et caractérisées par une instabilité chromosomique ainsi que par une hypersensibilité à certains agents physiques ou chimiques [17]. L'association d'un défaut de réparation de l'ADN lésé à la prédisposition au cancer fait de ces maladies à contrôle souvent multigénique de bons modèles pour examiner un ensemble de questions fondamentales en biologie cellulaire. Le clonage et la caractérisation des gènes impliqués devraient permettre non seulement d'améliorer le diagnostic et de détecter les porteurs hétérozygotes, mais également de mieux comprendre l'expression, la régulation et la coordination des différentes fonctions touchées.

Quand on fait le bilan de l'apport des mutants de réparation de Escherichia coli ou de levure dans notre compréhension des réseaux métaboliques impliqués dans la mutagenèse et la recombinaison génétique, on mesure à la fois leur immense utilité et l'ampleur de la tâche qui nous attend pour les cellules humaines

\section{TIRÉS À PART}

E. Moustacchi : institut Curie, biologie, 26, rue d'Ulm, 75231 Paris Cedex 05, France.

\section{RÉFÉRENCES}

1. Fanconi G. Familial constitutional panmyelocytopathy, Fanconi's anemia (FA). I : Clinical aspects. Semin Hematol 1967 ; 4 : 233-40. 2. Swift M. Fanconi's anaemia in the genetics of neoplasia. Nature $1971 ; 230: 370-3$.

3. Swift M, Caldwell RJ, Chase C. Reassement of cancer predisposition of Fanconi anemia heterozygotes. J Natl Cancer Inst 1980 ; 65 : 863-7.

4. Schroeder TM, Anschutz F, Knopp A Spontane chromosomen-aberrationen bei familiärer Panmyelopathie. Hum Genet 1964; 1 : 194-6.

5. Auerbach AD, Sagi M, Adler B. Fanconi anemia : prenatal diagnosis in 30 fetuses at risk. Pediatrics 1985 ; 76 : 794-800.

6. Dutrillaux B, Aurias A, Dutrillaux AM, Buriot D, Prieur M. The cell cycle of lymphocytes in Fanconi anemia. Hum Genet $1982 ; 62$ : 327-32

7. Schindler D, Kubbies M, Hoehn H, Schinzel A, Rabinovitch PS. Presymptomatic diagnosis of Fanconi's anaemia. Lancet 1985 ; i : 937.

8. Glanz A, Fraser FC. Spectrum of anomalies in Fanconi anaemia. $J$ Med Genet 1982 ; 19 : 412-6.

9. Duckworth-Rysiecki G, Cornish K, Clarke CA, Buchwald M. Identification of two complementation groups in Fanconi's anemia. Somat Cell Mol Genet 1985; 11 : 35-43.

10. Moustacchi E, Diatloff-Zito C. DNA semiconservative synthesis in normal and Fanconi anemia fibroblasts following treatment with 8-methoxypsoralen and near ultraviolet light or with X-rays. Hum Genet $1985 ; 70$ : 236-42.

11. Moustacchi E, Papadopoulo D, DiatloffZito C, Buchwald M. Two complementation groups of Fanconi's anemia differ in their phenotypic response to a DNA-crosslinking treatment. Hum Genet $1987 ; 75$ : 45-7.

12. Spivak G, Ganesan AK, Hanawalt PC, Enhanced transformation of human cells by UV-irradiated $\mathrm{pSV}_{2}$ plasmids. Mol Cell Biol $1984 ; 4: 1169-71$.

13. Van Duin M, Westerveld A, Hoeijmakers JHJ. UV stimulation of DNA-mediated transformation of human cells. Mol Cell Biol 1985 ; $5: 734-41$

14. Diatloff-Zito C, Papadopoulo D, Averbeck D, Moustacchi E. Abnormal response to DNA crosslinking agents of Fanconi anemia fibroblasts can be corrected by transfection with normal human DNA. Proc Natl Acad Sci USA 1986 ; 83 : 7034-8.

15. Rubin JS, Prideaux VS, Huntington FW, Dulhanty AM, Whitmore GF, Bernstein A. Molecular cloning and chromosomal localization of DNA sequences associated with a human DNA repair gene. Mol Cell Biol 1985 ; 5 : 398-405.

16. Westerfeld A, Hoeijmakers JHJ, Van Duin $M$ et al. Molecular cloning of a human DNA repair gene. Nature 1984 ; 310 : 425-9.

17. Hanawalt PC, Sarasin A. Cancer-prone hereditary diseases with DNA processing abnormalities. Trends In Genetics 1986; 2 : 124-9. 